\title{
Análisis del Desempeño de Sistema de Detección de Señal SSVEP Utilizando Clasificadores Árbol Simple y Máquina de Vectores de Soporte
}

\section{Performance Analysis of SSVEP Signal Detection} System Using Simple Tree and Support Vector Machine Classifiers

\author{
Alma Delia Corral Sáenz ${ }^{1}$ \\ adcorral@itchihuahua.edu.mx \\ Raúl Rangel González ${ }^{1}$ \\ rrangel@itchihuahua.edu.mx \\ Mario Ignacio Chacón Murguía \\ mchacon@ieee.org
}

\footnotetext{
${ }^{1}$ Laboratorio de Sistemas de Percepción Visual con Aplicaciones en Robótica, Tecnológico Nacional de México/ I.T. Chihuahua, México.
} 
Resumen: Un tipo de interfaces cerebro-computadora $(\mathrm{BCl})$ son los sistemas que interpretan patrones de actividad cerebral para facilitar a personas con discapacidad motora o del habla, la comunicación o el control de dispositivos. Una forma de comunicación exitosa han sido los deletreadores basados en potenciales evocados de estado estable (SSVEP por sus siglas en inglés) que parten de la generación de estímulos visuales para provocar determinada actividad cerebral, registrarla e interpretarla mediante algoritmos de clasificación. Dentro de estos sistemas falta realizar un análisis para determinar un clasificador que tenga una relación tiempo de procesamiento y desempeño eficientes para aplicaciones en entornos controlados. En este trabajo se parte de la generación y registro de señales SSVEP, para realizar un análisis de desempeño de los clasificadores de árbol simple ST, y de máquinas de vectores de soporte SVM, los cuales son algoritmos de baja complejidad computacional encontrados en los métodos de análisis de SSVEP. De acuerdo a los resultados obtenidos, el porcentaje promedio de acierto del ST para una señal de 10 s fue de $84.58 \%$ para el canal O1 y un $78.75 \%$ para el O2; mientras que el del SVM fue $78.33 \%$.

Palabras clave: $\mathrm{BCl}$, señal EEG, SSVEP, ST, SVM.

Abstract: One type of Brain computer interfaces are the systems that interpret brain activity patterns to assist people with motor or speech disability in communication or control devices tasks. A successful manner of communication is the use of spellers based on steady state visually evoked potentials (SSVEP) which are generated from visual stimulus. These stimuli are later registered and interpreted through classifiers algorithms. However, there is a lack of analysis in these systems that consider processing time and efficient performance for applications in controlled environment. In this work, SSVEP signals are generated and registered to analyzed the performance of simple tree (ST) and support vector machine (SVM) classifiers, which are low computational complexity algorithms found in methods of SSVEP analysis. According to the results, the average percentage of success of the ST for a $10 \mathrm{~s}$ signal was $84.58 \%$ for the $\mathrm{O} 1$ channel and $78.75 \%$ for the O2 channel; while for the SVM was $78.33 \%$.

Keywords: $\mathrm{BCI}, \mathrm{EEG}$ signal, SSVEP, Simple tree, Support vector machines. 


\section{Introducción}

El desarrollo o mejoramiento de esquemas de comunicación para personas que presentan discapacidad motora y del habla ha cobrado mayor interés desde el surgimiento de las interfaces cerebro-computadora. Los deletreadores basados en potenciales evocados han sido una de las estrategias que esta tecnología ha propiciado. Estas interfaces de comunicación presentan al usuario 0 paciente un estímulo que provoca cambio en actividad cerebral y es medido y registrado mediante señales de electroencefalografía (EEG) que posteriormente son procesadas, caracterizadas e interpretadas para dar un significado al estímulo detectado. Los SSVEP son potenciales evocados que han sido utilizados de forma exitosa para estos fines de comunicación y se basan en la generación de un estímulo a una frecuencia determinada que provoca que en la actividad cerebral se refleje la misma frecuencia. SSVEP es un potencial evocado muy común en la literatura ya que muestra un comportamiento estable y no requiere de entrenamiento en el usuario final (Lim, Lee, Hwang, Kim, \& Im, 2015) (Zhang, Yu, Yin, \& Zhou, 2016).

La generación del estímulo SSVEP se realiza mediante figuras $u$ objetos que parpadean a cierta frecuencia, por ejemplo, rectángulos (Zhanga, Zhou, Jin, Wanga, \& Cichockib, 2015), teclados (Lim, Lee, Hwang, Kim, \& Im, 2015), dibujos (Zhao, y otros, Junio 2017), dameros (Funase, Wakita, Ita, \& Takumi, 2016), etc. La adquisición de las señales SSVEP es mediante electrodos colocados de acuerdo al sistema internacional 10-20 y en diversos trabajos se presentan diferentes configuraciones de electrodos para obtener la señal SSVEP. Sin embargo, de acuerdo a (Lee, Lin, Cherng, \& Ko, 2016), con el uso de los electrodos $O_{1}$ y $O_{2}$ es suficiente para adquirir correctamente la señal SSVEP. El análisis y procesamiento de las señales SSVEP se hace tradicionalmente extrayendo e interpretando características en el dominio de la frecuencia utilizando métodos como análisis del espectro de potencia (Zhanga, Zhou, Jin, Wanga, \& Cichockib, 2015) (Lee, Lin, Cherng, \& Ko, 2016), análisis canónico de correlación (Zhanga, Zhou, Jin, Wanga, \& Cichockib, 2015) (Lee, Chuang, Yeng, Chen, \& Lin, Dic 2017), análisis de componentes independientes (Nakanishi, Yijun, Hsu, Wang, \& Jung, sep 2017), redes neuronales artificiales (Turnip, Suhendra, K, \& Sihombing, 2017) o tensores (Ji, y otros, 2016). Entre las aplicaciones del análisis de la señal SSVEP se encuentran deletreo con caracteres alfanuméricos (Lim, Lee, Hwang, Kim, \& Im, 2015) (Yin, Zhou, Jiang, Yu, \& Hu, junio 2015) (Tang, Wang, Dong, Pei, \& Chen, 2017), marcación de números telefónicos (Cheng, Gao, Gao, \& Xu, oct 2002), robótica (Zhao, y otros, Junio 2017), rehabilitación (Zhao, Chu, Han, \& Zhang, julio 2016) y aplicaciones enfocadas a estudiar el funcionamiento del ojo y el sistema visual humano como en (Cotrina, Benevides, Castillo-Garcia, \& Benevides, julio 2017) (Maye, Zhang, \& Engel, julio 2017). 
De las aplicaciones mencionadas, los deletreadores han sido las más recurridas ya que mediante la selección de símbolos se pueden trasmitir diferentes mensajes que pueden ser utilizados en múltiples aplicaciones. Los deletreadores han utilizado distintas figuras y objetos para generar el estímulo, pero uno que ha sido poco explorado es el damero, una figura similar a un tablero de ajedrez que de acuerdo a (Funase, Wakita, Ita, \& Takumi, 2016), estimula efectivamente la corteza visual. Esta área de oportunidad motiva a proponer en este trabajo un método novedoso para detectar una señal SSVEP generada con un deletreador que utiliza dameros como el estímulo que provoca en la actividad cerebral el patrón de determinada frecuencia. Para ello, el método propuesto contempla un protocolo de adquisición de señales, realiza un análisis en el dominio de la frecuencia para el preprocesamiento y extracción de características, clasifica mediante dos modelos: máquinas de vectores de soporte y árbol simple; interpreta a cuál carácter de la interfaz corresponde y realiza una toma de decisión que conducirá al carácter elegido.

El resto del artículo se presenta de la siguiente manera: en la sección 2 se plantea el método propuesto, en la sección 3 se describe el deletreador desarrollado, en la sección 4 se muestran los resultados y en la sección 5 se presentan las conclusiones.

\section{Método Propuesto}

La Figura 1 muestra el esquema general del método propuesto que consta de las etapas de adquisición, preprocesamiento, extracción de características, clasificación de la señal SSVEP e interpretación en la interfaz. Todas estas etapas se describen en las siguientes subsecciones.

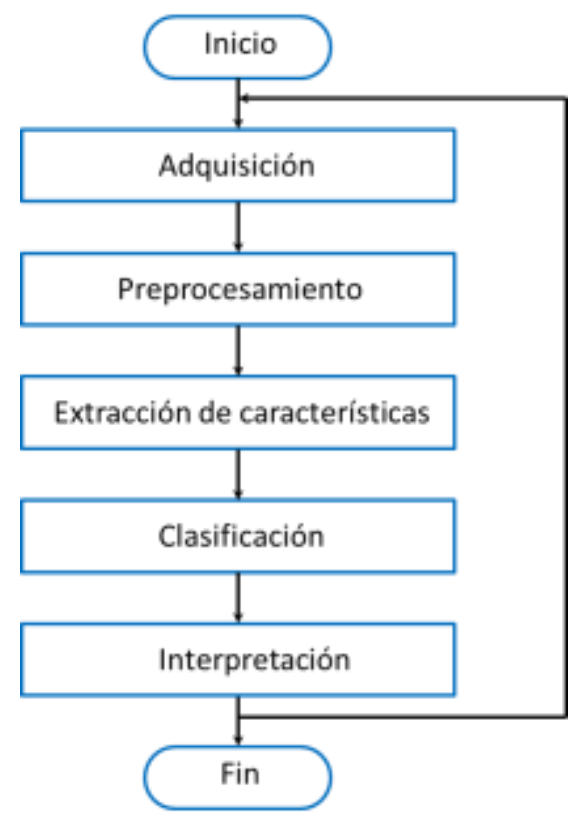

Figura 1. Esquema general del método propuesto. 


\subsection{Adquisición de señal}

En la etapa de adquisición de la señal, se genera el estímulo SSVEP mediante dameros que parpadean a determinada frecuencia, creando la ilusión de que los cuadros están cambiando de posición (Zhang, Yu, Yin, \& Zhou, 2016). Los dameros empleados se componen de patrones blanco y negro en $4 \times 4$ cuadros internos y un punto rojo en el centro para que el usuario enfoque su mirada. En la Figura 2 se muestra un damero con las dimensiones empleadas.

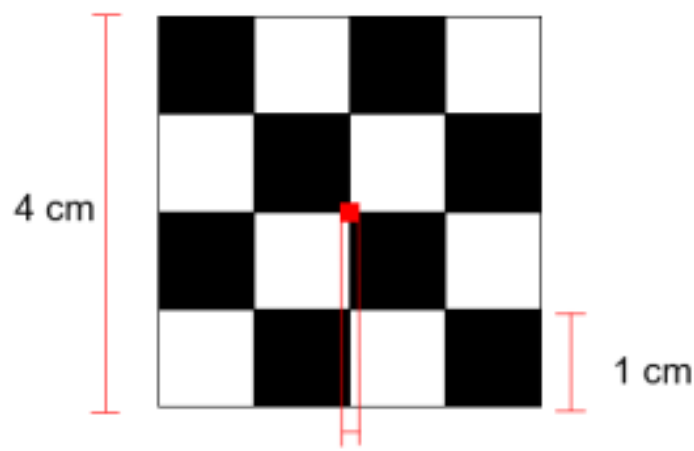

$2 \mathrm{~mm}$

Figura 2. Damero con patrón de colores blanco y negro con punto rojo en el centro para enfocar la mirada.

Las especificaciones en la generación de la señal se basan en las dimensiones de los cuadros internos del damero, distancia entre el damero y el sujeto, la frecuencia de parpadeo y las condiciones del entorno. Las dimensiones de los cuadros internos se determinaron al fijar un ángulo de visión de 1 grado que debe tener el sujeto de pruebas al mirar el cuadro interno del estímulo a una distancia común de $55 \mathrm{~cm}$ al mirar la pantalla de una computadora. El ángulo de visión de 1 grado es usual en la generación de potenciales evocados visuales en procedimientos clínicos (Trujillo \& Borrego, 1985). La dimensión de los cuadros internos del damero se define por:

$$
\operatorname{dim}=2 *\left(D * \arctan \left(\frac{\theta}{2}\right)\right)
$$

donde $D$ es la longitud en centímetros entre el ojo del sujeto y la pantalla donde están los estímulos y $\theta$ es el ángulo de visión que se desea obtener. Para seleccionar los valores de frecuencias de parpadeo se realizaron dos consideraciones: que una frecuencia no debe ser múltiplo de otra para evitar falsos positivos por armónicos, y que deben evitarse frecuencias dentro de la banda alfa, especialmente entre los 10 y $11 \mathrm{~Hz}$ debido a que en la región occipital del cerebro se presenta gran actividad de este tipo al cerrar y abrir los ojos y la información generada por esta actividad podría presentar una mayor 
amplitud que la que se genera por un SSVEP. Las frecuencias de parpadeo entonces fueron definidas mediante la fórmula:

$$
F_{p}=\frac{F_{r}}{M}
$$

donde $F_{r}$ se refiere a la frecuencia de actualización de la pantalla y $M$ es un número entero, de manera que las frecuencias de parpadeo obtenidas se muestran en la Tabla 1:

Tabla 1. Frecuencias de parpadeo

\begin{tabular}{|c|c|}
\hline $\begin{array}{c}\text { Número entero } \\
M\end{array}$ & $\begin{array}{c}\text { Frecuencia de } \\
\text { parpadeo } \\
F_{p}\end{array}$ \\
\hline 10 & $6 \mathrm{~Hz}$ \\
\hline 9 & $6.6 \mathrm{~Hz}$ \\
\hline 8 & $7.5 \mathrm{~Hz}$ \\
\hline 7 & $8.57 \mathrm{~Hz}$ \\
\hline
\end{tabular}

Al momento de generar la señal, ciertas condiciones del entorno se mantuvieron bajo control para facilitar que las características de las muestras fueran consistentes y que los cambios producidos en ellas fueran inducidos puramente por el sujeto de pruebas como respuesta al estímulo visual del damero y no por condiciones externas. Las variables bajo control son:

Iluminación. Se debe contar con una iluminación baja (Ding, Sperling, \& Srinivasan, 2006). Se obtuvieron las muestras en habitación con 4 luxes en promedio.

Luces parpadeantes. No deben existir dentro del campo de visión del usuario para evitar distracciones.

Elementos distractores. No deben existir dentro del campo de visión del usuario (Bondre \& Kapgate, 2015).

Postura. Se sugiere tener una postura cómoda y similar en todos los casos (Bondre \& Kapgate, 2015). Los sujetos de prueba utilizaron una silla con descansa brazos para tener una postura uniforme.

Temperatura. Debe ser agradable para el sujeto de pruebas. Se mantuvo un rango entre los 22 y $26^{\circ} \mathrm{C}$ durante la toma de muestras. 
Una vez definidas las condiciones en la selección del estímulo, fue seleccionada la diadema Emotiv EPOC $+{ }^{\circledR}$ como el dispositivo para registrar la actividad cerebral. La diadema tiene las siguientes características:

- Es un dispositivo comercial y accesible en costo para cualquier persona.

- Ofrece frecuencia de muestreo de $128 \mathrm{~Hz}$, suficiente para muestrear la frecuencia de parpadeo más alta de los dameros que es de $8.57 \mathrm{~Hz}$.

- Cuenta con 14 electrodos para adquisición de señales de EEG, de los cuales fueron seleccionados el $\mathrm{O}_{1}$ y $\mathrm{O}_{2}$ que se posicionan en el lóbulo occipital, área cerebral en donde se encuentra la corteza visual, activada por el estímulo del damero. De acuerdo a (Lee, Lin, Cherng, \& Ko, 2016), estos electrodos son suficientes para la obtención de las señales SSVEP.

- Proporciona datos crudos de las señales EEG.

- Tiene resolución mayor a $10 \mu \mathrm{V}$, que es el rango de amplitud que tienen los potenciales evocados (Abbasi, Gaume, Francis, Dreyfus, \& Vialatte, 2015).

- Tiene un rango dinámico mayor $\mathrm{a} \pm 10 \mu \mathrm{V}$ para no tener perdida de información.

La lectura de los electrodos $O_{1}$ y $O_{2}$ se realiza mediante el software TestBench. La frecuencia de muestreo es de $128 \mathrm{~Hz}$ y fueron descartadas las señales donde se presentaron artefactos por desconexión de electrodos, es decir, solo se consideraron aquellas en las que ambos electrodos hicieron un registro efectivo.

La adquisición de señales fue realizada con 3 sujetos de prueba durante un evento llamado sesión. La sesión se refiere a un lapso de 40 minutos en el que se adquirieron 120 señales de 20 segundos cada una. Con los primeros datos de las señales de $20 \mathrm{~s}$ se crean otras 120 señales de una ventana de $10 \mathrm{~s}$ y 120 señales de una ventana de $5 \mathrm{~s}$, con lo que al final se tienen para análisis 360 señales de cada sujeto. Las señales adquiridas se definen en este artículo como $x_{p}(n)$, donde $n$ es el índice de tiempo y $p$ se refiere a los canales definidos por los electrodos $\mathrm{O}_{1}$ y $\mathrm{O}_{2}$.

\subsection{Preprocesamiento de la señal}

Una vez adquirida la señal por ambos electrodos o canales, a cada una de ellas se le aplicó la transformada discreta de Fourier (DFT por sus siglas en inglés) definida por:

$$
X_{p}(k)=\sum_{n=1}^{N-1} x_{p}(n) e^{-j \frac{2 \pi k n}{N}}, \quad k=1, \ldots, N \quad p=\left\{O_{1}, O_{2}\right\}
$$


donde $k$ es el índice de frecuencia, $N$ el tamaño de la señal. Luego se realiza un ajuste para eliminar la mitad redundante y el escalamiento en $N / 2$ mediante:

$$
Y_{p}(k)=\left|2 X_{p}(k) / N\right| \quad \text { para } \quad 1 \leq k \leq N / 2+1 .
$$

\subsection{Extracción de características}

Para este trabajo, se consideró que la característica más relevante que se puede obtener de la actividad cerebral al presentarse el estímulo SSVEP es la componente de frecuencia con mayor amplitud en la señal. Primero se acota $Y_{p}(k)$ a la banda entre $5.6 \mathrm{~Hz}$ y $9 \mathrm{~Hz}$ para que contenga solo la banda de las 4 frecuencias de parpadeo definidas con (2). Para ello, se calcula la resolución de la frecuencia en $\mathrm{Hz}$ mediante:

$$
\operatorname{Res}=\frac{\mathrm{Fs}}{\mathrm{N}}
$$

donde $F_{s}$ es la frecuencia de muestreo. Luego se recorta la señal $Y_{p}(k)$ de la siguiente forma:

$$
\mathrm{Y}_{j p}(\mathrm{k})=\left\{\mathrm{Y}_{p}(\mathrm{k}) \mid \frac{5.6}{\operatorname{Re} s} \leq \mathrm{k} \leq \frac{8.8}{\operatorname{Re} s}\right.
$$

donde el 5.6 y el 8.8 se refieren a los límites inferior y superior de las frecuencias en las cuales se puede presentar el estímulo. Una vez obtenido el vector $Y_{f c}(k)$ acotado dentro de las frecuencias de interés, se procede a encontrar los índices de las amplitudes máximas de las frecuencias en los canales $O_{1}$ y $O_{2}$ definidas como $k m a x_{i j}$, donde $i=20 \mathrm{~s}$, $10 \mathrm{~s}$, $5 \mathrm{~s}$ y $j=\left\{O_{1}, O_{2}\right\}$, luego se obtienen los valores de frecuencia que les corresponden:

$$
f_{i j}=k \max _{i j} \text { Res } \quad \text { para } i=20 \mathrm{~s}, 10 \mathrm{~s}, 5 \mathrm{~s} ; j=\left\{\mathrm{O}_{1}, \mathrm{O}_{2}\right\}
$$

mismos que serán definidos como características de frecuencia. Este procedimiento se realiza a cada muestra de las ventanas de 20,10 y $5 \mathrm{~s}$ y se guardan en un vector en el orden siguiente: los primeros dos elementos corresponden a las características obtenidas cuando se aplicó el estímulo de 6 $\mathrm{Hz}$, los siguientes corresponden al de $6.6 \mathrm{~Hz}$, los siguientes al de $7.5 \mathrm{~Hz}$ y los últimos al de $8.57 \mathrm{~Hz}$. Esto define el siguiente vector de características:

$$
\text { feat }_{i}=\left\{f_{i O 1}, f_{i O 2}\right\}
$$


En la Figura 3 se muestra el espacio de características de 120 señales de la ventana de $20 \mathrm{~s}$ para el sujeto 1 , siendo el eje horizontal el valor de $f_{01}$ y el eje vertical el valor de $f_{02}$. Los ejes están en escala de Hertz. Con la gráfica se pretende mostrar que tanto el valor de $f_{01}$ como el de $f_{02}$ son similares en la mayoría de los casos y están lo suficientemente separadas entre clases. En la gráfica se pueden observar grupos definidos por $f_{01}$ y $f_{02}$ en los que los valores de ambos elementos no son iguales, lo que hace que estos vectores se separen del grupo donde se encuentra su respectiva clase, esto pasa generalmente por no seguir correctamente el protocolo de toma de muestras y se generan frecuencias con mayor amplitud que las del SSVEP.

Las características que se muestran en la Figura 3 son 120 en total, pero se encuentran sobrepuestas, ya que varias sesiones generaron los mismos valores tanto en el electrodo $O_{1}$ como en el $O_{2}$, por lo cual aparecen en el mismo punto.

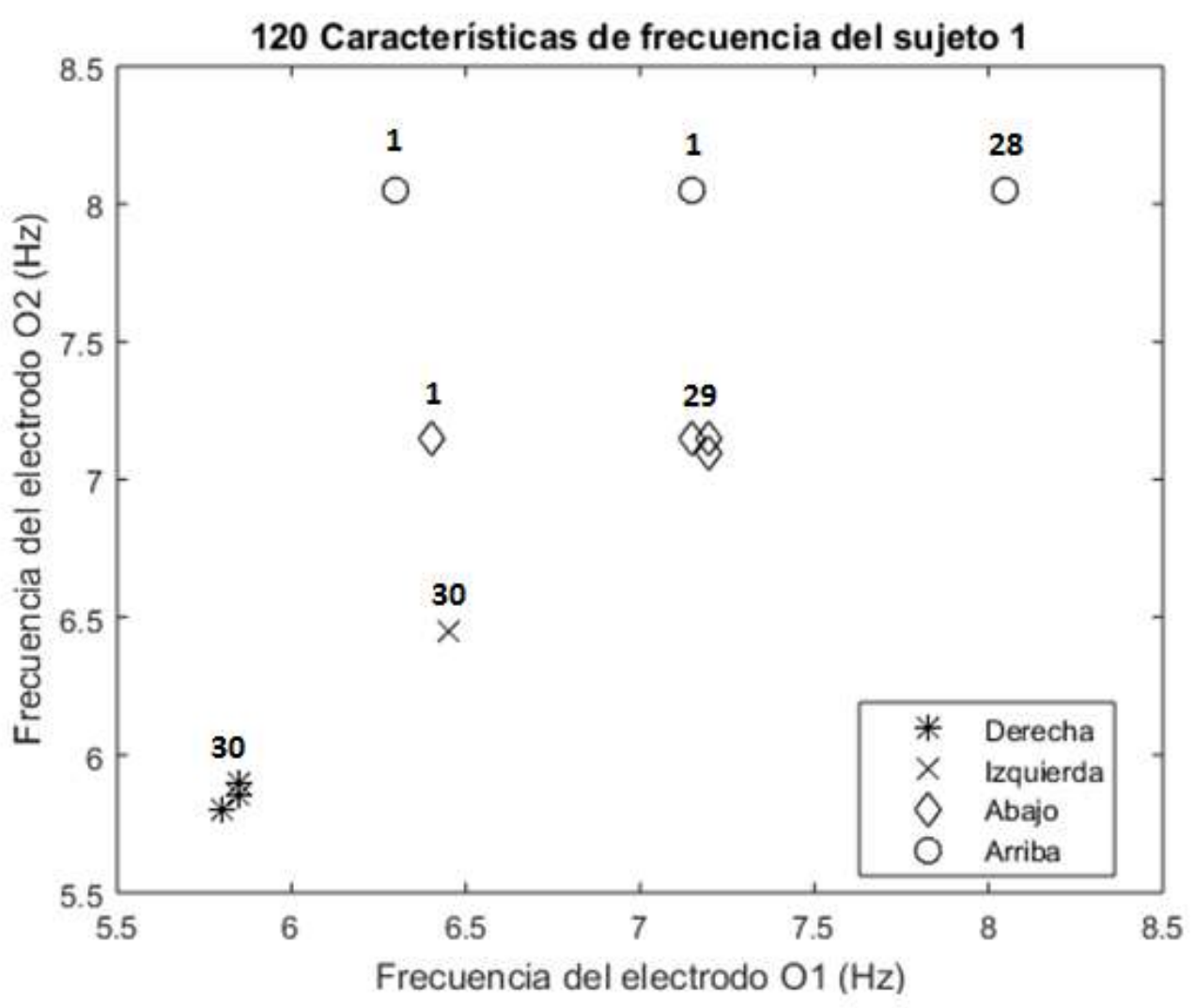

Figura 3. Gráfica de las características de frecuencia de los electrodos $O_{1}$ y $O_{2}$ del sujeto 1.

\subsection{Clasificación}

En la literatura, existen varios tipos de clasificadores para estudiar el fenómeno de SSVEP (da Cruz, Wan, Wong, \& Cao, 2015) (Shyam Prasad, et al., 2017). Para seleccionar el clasificador con mejor rendimiento, se realizó un análisis 
estadístico de los clasificadores SSVEP que se reportan en la literatura y con base en las conclusiones de éste, se optó por seleccionar los clasificadores simple tree (ST) y support vector machine (SVM) debido que ambos tienen una velocidad de predicción rápida, un uso de memoria bajo para el ST y medio para el SVM y una fácil interpretación. El uso del ST es útil cuando se trabaja con un solo electrodo y el SVM cuando se trabaja con la información de ambos electrodos. Para generar el conjunto de datos de entrenamiento de ambos clasificadores, se obtuvieron 120 pruebas con un sujeto y para generar el conjunto de datos de prueba, se obtuvieron 360 pruebas con tres sujetos. Las clases se refieren al damero que fue seleccionado según se muestra en la Tabla 2.

Tabla 2. Nombres de las clases según la posición de los dameros.

\begin{tabular}{|c|l|l|}
\hline $\begin{array}{l}\text { Rangos de } \\
\text { frecuencia }\end{array}$ & $\begin{array}{l}\text { Posición del } \\
\text { damero en la } \\
\text { interfaz }\end{array}$ & $\begin{array}{l}\text { Nombre de la } \\
\text { clase }\end{array}$ \\
\hline $5.6-6.15$ & Parte derecha & Derecha \\
\hline $6.175-6.8$ & Parte izquierda & Izquierda \\
\hline $6.775-7.6$ & Parte inferior & Abajo \\
\hline $7.6-8.8$ & Parte superior & Arriba \\
\hline
\end{tabular}

\subsubsection{Clasificador ST}

Para la creación del clasificador ST se realizó lo siguiente: se extrajo del valor esperado dentro de cada clase y se crearon de 3 nodos, cada uno posicionado en el punto medio de las clases adyacentes: $6.15 \mathrm{~Hz}, 6.8 \mathrm{~Hz}$ y $7.6 \mathrm{~Hz}$. El algoritmo a utilizar es el ID3 (Mitchell, 1997) que hace uso de la ganancia de información y la entropía $E$. La ganancia se utiliza para saber el orden de los nodos dentro del árbol y la entropía para saber si es un nodo es final o si se dividirá aún más. La entropía se calcula de la siguiente manera:

$$
E(S)=-\sum_{z \in Z} p(z) \log _{2}(p(z))
$$

donde $S$ es el conjunto de datos que contienen las frecuencias de las clases (de $5.5 \mathrm{a} 8.8 \mathrm{~Hz}$ ), $Z$ el conjunto de clases que se pueden encontrar en $S$ y $p(z)$ la probabilidad de obtener un elemento de la clase $z$ en el conjunto $S$. En este caso $z$ se refiere a la frecuencia SSVEP que se debe detectar. Cuando el valor de la entropía sea igual a cero indicará que todos los datos del conjunto $S$ de elementos pertenecen a la misma clase, por lo que el nodo evaluado será el último. El cálculo de la ganancia de información se realiza de la siguiente forma. 


$$
G(S, A)=E(S)-\sum_{i \in T} p(t) E(t)
$$

donde $T$ se refiere a los subconjuntos de datos que se crean al dividir el conjunto $S$ por la condición que impone el atributo $A, p(t)$ la probabilidad de obtener un elemento del subconjunto $t$, el cual se refiere al rango de frecuencias que definen cada clase y $E(t)$ la entropía del subconjunto $t$. El atributo que se escoge para dividir a un nodo es el que mayor ganancia de información tenga.

Debido a que los valores del sujeto de prueba son distribuidos por partes iguales dentro de cada clase el valor de ganancia de información va a ser igual para cada atributo, por lo que el orden de división de los nodos se escoge por su valor numérico de forma ascendente. La entropía fue indicando cuando detener la división de los nodos. El diagrama del árbol generado se muestra en la Figura 4, donde $f_{o x}$ indica que se trata de una característica de frecuencia de cualquiera de los dos electrodos.

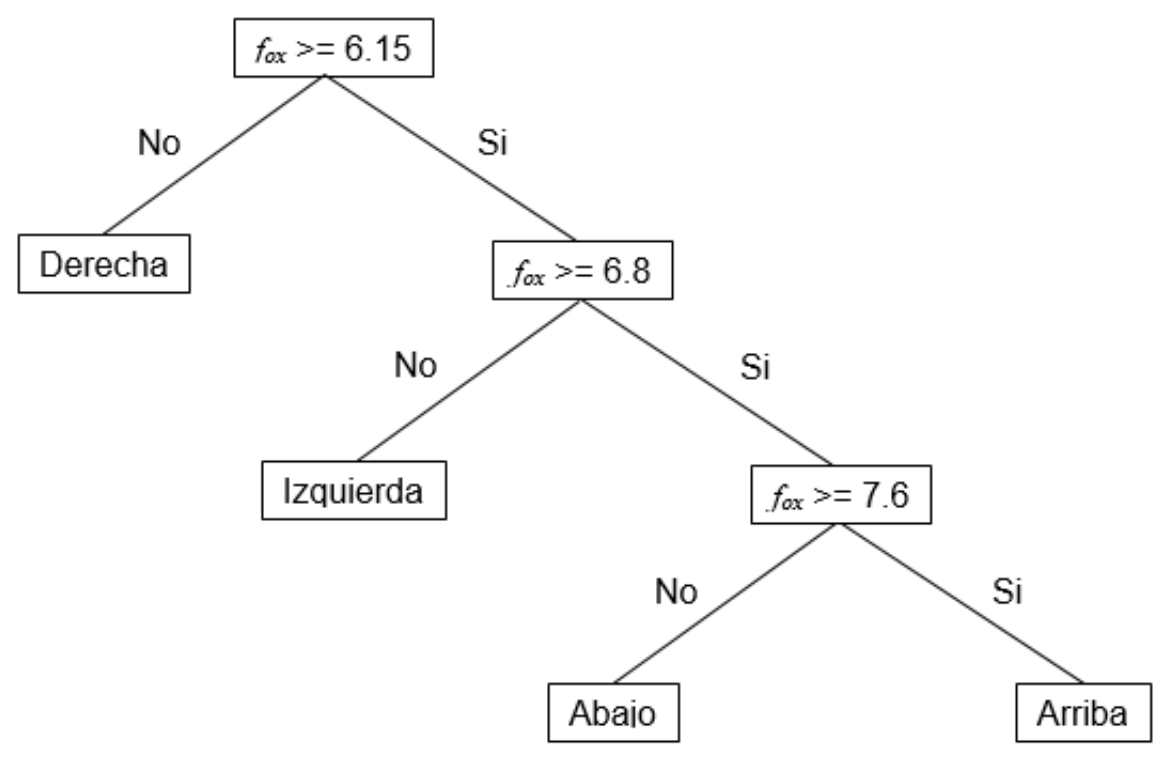

Figura 4. Clasificador Simple Tree.

Una vez generado el árbol de decisión con los 3 atributos se implementó un algoritmo basado en condicionales para evaluar las características de feat ${ }_{i}$ en el orden en el que se encuentran los atributos hasta encontrar la clase a la que pertenecen. Este algoritmo se define de la siguiente forma: 
Si feat ${ }_{i}$ es menor a 6.15

Establecer la clase a la que pertenece feat ${ }_{i}$ como "Derecha";

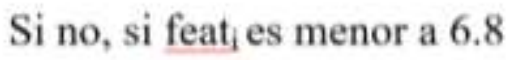

Establecer la clase a la que pertenece feat ${ }_{i}$ como "Izquierda";

Si no, si feat ${ }_{i}$ es menor a 7.6

Establecer la clase a la que pertenece feat; como "Abajo";

Si no, si feat ${ }_{i}$ es igual o mayor a 7.6

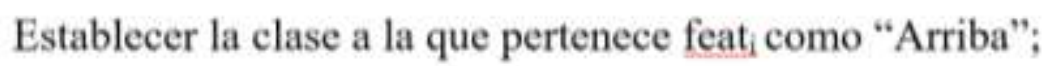

\subsubsection{Clasificador SVM}

En este caso, se generó un SVM para definir un conjunto de funciones discriminantes lineales (FDL) que definan un clasificador multiclase. Cada FDL separa un espacio geométrico de características en 2 clases mediante un hiperplano que generalmente es $H\left(f_{e a t} t_{i}=0\right.$. En este trabajo fue utilizada una función de Matlab $^{\circledR}$ para generar un conjunto de FDL para los casos multiclases. Esta función pide como parámetros de entrada las características feat $t_{i}$ también la clase a la que pertenecen. Por defecto la función regresa un clasificador SVM lineal del tipo uno contra uno; este tipo quiere decir que se crea una FDL por cada par de clases. El número de FDL se define de la siguiente forma:

$$
n_{F D L}=n_{c}\left(n_{c}-1\right) / 2
$$

donde $n_{c}$ es el número de clases, así que para manejar 4 clases son necesarios 6 FDL. Con las 6 FDL creadas con la función fitecoc() se procede a generar las ecuaciones que los definen. De manera general la ecuación de una FDL es la siguiente:

$$
H_{l}\left(f_{\text {caract }}\right)=\beta_{O 1} f_{i O 1}+\beta_{O 2} f_{i O 2}+b, \quad l=1, \ldots, 6
$$

donde $\beta_{01}$ y $\beta_{02}$ son los pesos para formar la pendiente del hiperplano, $b$ el offset del hiperplano y I un índice por FDL. A continuación se muestran las ecuaciones de cada FDL. 


$$
\begin{aligned}
& H_{1}\left(f_{\text {caract }}\right)=-1.6917 f_{\text {io1 }}-1.6417 f_{\text {io2 }}+20.5 \\
& H_{2}\left(f_{\text {carract }}\right)=-0.55 f_{\text {iol } 1}-1.25 f_{i o 2}+11.5612 \\
& H_{3}\left(f_{\text {caract }}\right)=-0.1865 f_{\text {iol }}-0.8912 f_{\text {io } 2}+7.3492 \\
& H_{4}\left(f_{\text {caract }}\right)=-1.1036 f_{i o 1}-1.7536 f_{i o 2}+19.4286 \\
& H_{5}\left(f_{\text {caract }}\right)=4.5861 * 10^{-4} f_{\text {lo1 }}-1.2505 f_{\text {io } 2}+9.0628 \\
& H_{6}\left(f_{\text {caract }}\right)=2.924 * 10^{-4} f_{\text {io1 } 1}-2.2219 f_{\text {io2 }}+16.8846
\end{aligned}
$$

Los hiperplanos son válidos para las señales en cualquier ventana de tiempo: 20, 10 y 5s. Para mostrar los hiperplanos de cada FDL se iguala a cero la ecuación y se toma $F_{i 01}$ para el eje horizontal y $F_{i 02}$ para el eje vertical. En la Figura 5 se puede observar cada uno de los hiperplanos, teniendo a la izquierda el nombre del FDL al que corresponde cada uno.

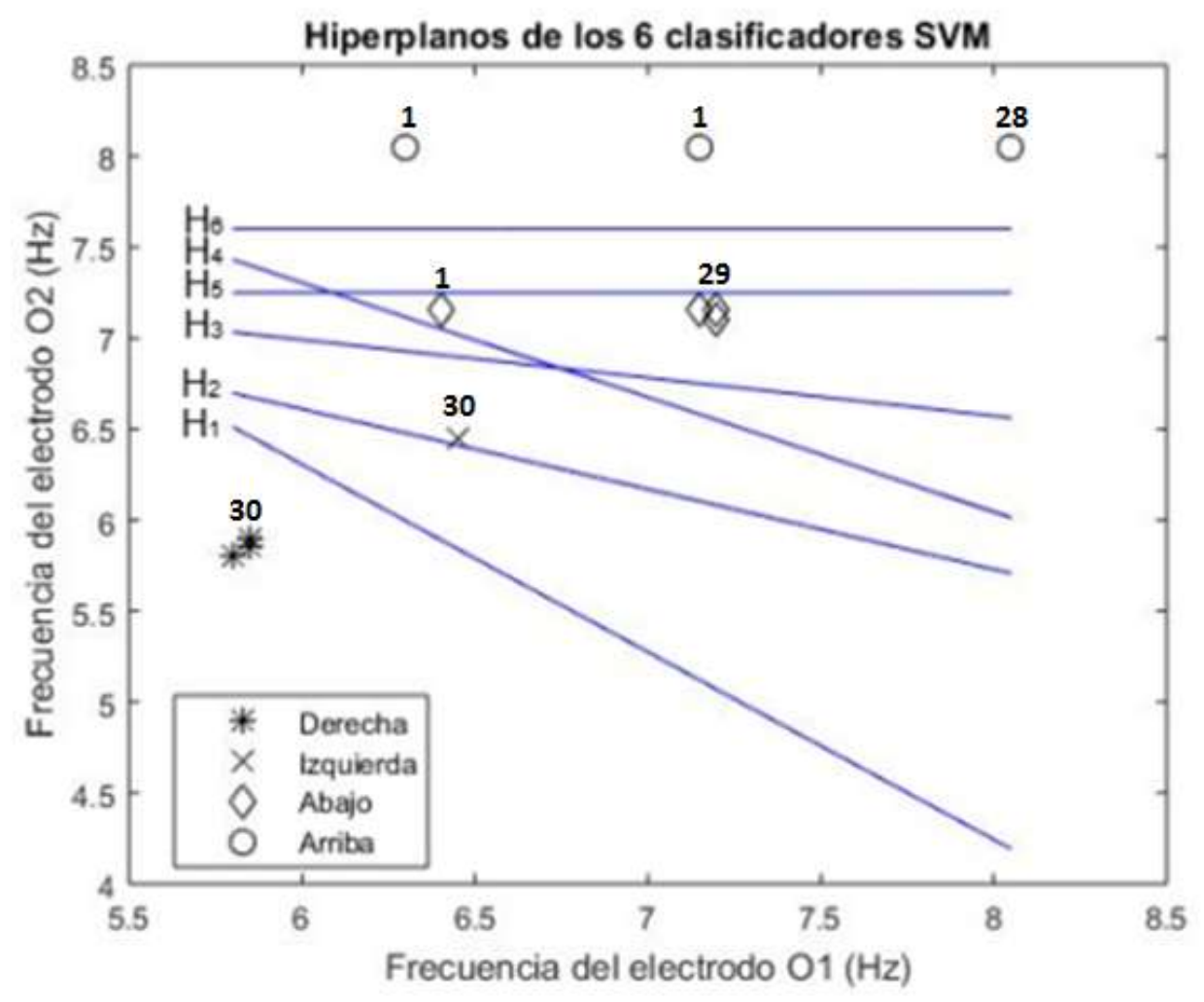

Figura 5. Hiperplanos de los 6 clasificadores SVM.

\subsection{Interpretación}

El último paso en la creación de un sistema $\mathrm{BCl}$ es la interpretación que se le da a la señal clasificada dentro una aplicación. En este trabajo la aplicación consiste en un deletreador para personas que carecen de movilidad y de la 
capacidad del habla. Para el deletreador se desarrollaron dos interfaces: una creada en Visual Studio usando el lenguaje de programación C\# y otra en Matlab. La interfaz de C\# es con la que interactúan directamente los sujetos de prueba; es la que se utiliza en la sesión para la toma de muestras, en la cual se generan los estímulos para evocar el SSVEP. La interfaz de Matlab es un formulario para indicar el modelo del clasificador que se va a usar y seleccionar las muestras que serán procesadas. Por ser un sistema $\mathrm{BCl}$ fuera de línea, esta interfaz se utiliza solo después de tomar las muestras. Ambas interfaces completan la aplicación. A continuación, se describe cada una de ellas.

\subsubsection{Deletreador: interfaz gráfica para toma de muestras}

La interfaz gráfica de usuario de Visual Studio fue programada en lenguaje C\#. La frecuencia de parpadeo de los estímulos fue generada usando temporizadores para que cada estímulo tuviera su propia frecuencia y no se interfirieran unos con otros. Los temporizadores tienen los siguientes retardos: 117 milisegundos para la frecuencia de $8.57 \mathrm{~Hz}, 133$ milisegundos para $7.5 \mathrm{~Hz}$, 150 milisegundos para $6.6 \mathrm{~Hz}$ y 167 milisegundos para $6 \mathrm{~Hz}$. En la Figura 6 se pueden ver los dameros que se encuentran en los 4 extremos de la pantalla y que mostrarán los estímulos a una frecuencia dada; el damero de la derecha tendrá una frecuencia de $6 \mathrm{~Hz}$, el de la izquierda de $6.6 \mathrm{~Hz}$, el de abajo $7.5 \mathrm{~Hz}$ y el de arriba $8.57 \mathrm{~Hz}$.

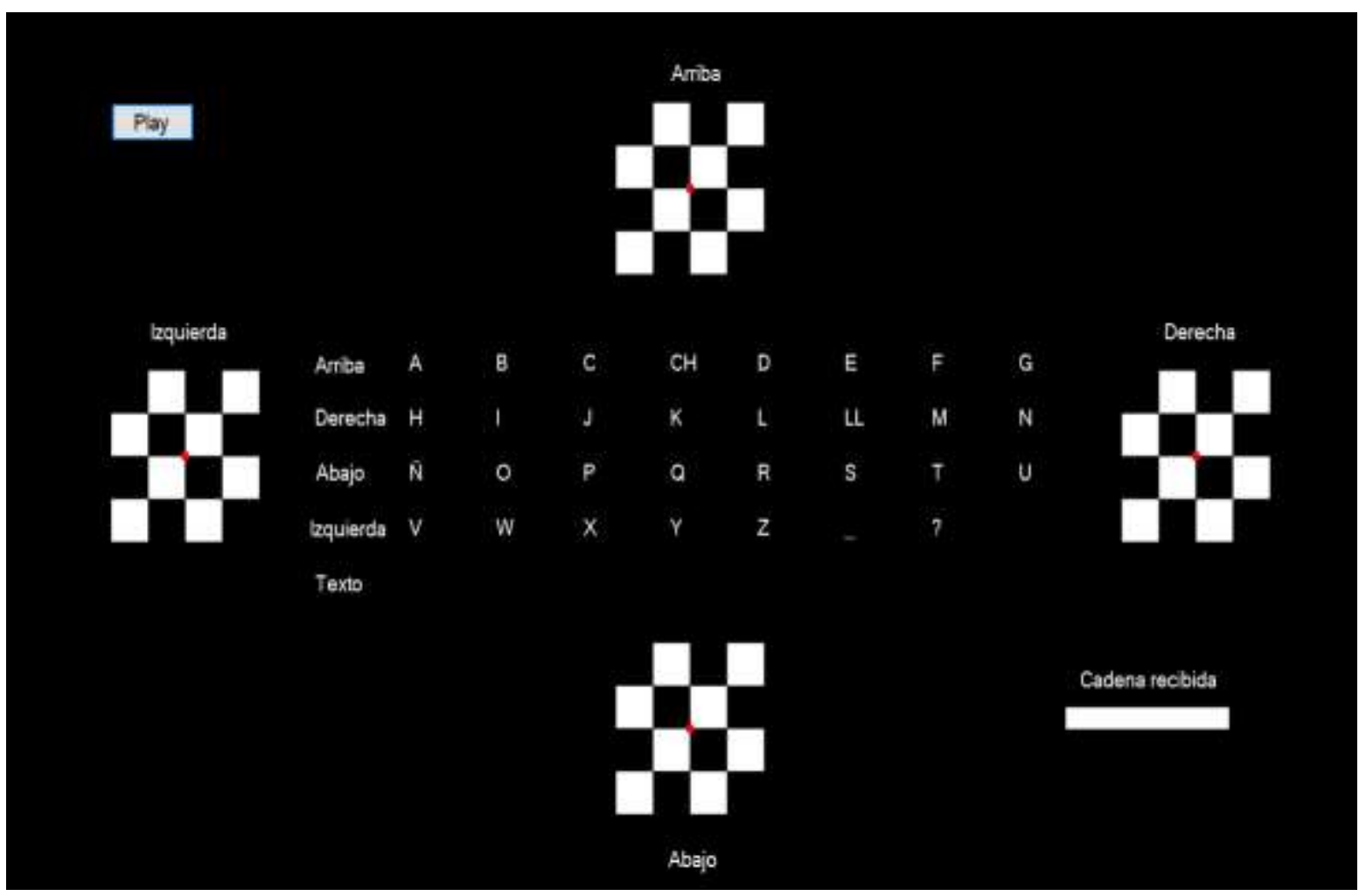

Figura 6. Interfaz gráfica para toma de muestras. 
La interfaz maneja un componente para configurar un puerto serial con los mismos valores que en Matlab y que se muestran a continuación:

- Tasa de baudios de 9600

- Tamaño del paquete de datos de 8 bits

- Sin bit de paridad

- 1 bit de parada

- Sin control de flujo

- Se usa un puerto de comunicación diferente entre Matlab y Visual Studio

El abecedario que se encuentra en el centro de la pantalla se creó con el uso de una caja de texto en la cual se escribieron las letras con espacios horizontales de una tabulación y verticales de 2 renglones. De este abecedario es donde se van a escoger las letras para deletrear, siendo el último componente necesario para la creación del deletreador. El abecedario cuenta con 27 letras 2 dígrafos 2 símbolos y la opción de borrar, en total son 32 opciones a elegir las cuales están divididas en 4 reglones. Para escoger una letra se elige el renglón en donde se encuentra la opción, se observa el estímulo correspondiente que hace que desaparezcan los demás renglones y las letras del elegido se redistribuyen en los 4 renglones y así sucesivamente hasta elegir la letra en 3 iteraciones. El sujeto simplemente tiene que aprenderse estos pasos para poder comunicarse con el mundo exterior, siendo esto una alternativa para poder expresar sus necesidades.

\subsubsection{Deletreador: interfaz de modelo de clasificador}

Para la ejecución de la aplicación es necesario definir ciertos parámetros como: el tipo del clasificador a utilizar, la ventana de datos que se extraerá de una señal, el sensor del cual se extraerán las características en caso de usar ST. También por ser un sistema $\mathrm{BCl}$ fuera de línea se requiere ingresar la señal que va a ser procesada. También es útil poder visualizar la clasificación de la señal y su representación en la frecuencia. Todo lo mencionado anteriormente se encuentra dividido en 3 secciones en la interfaz de control en Matlab que se muestra en la Figura 7. 


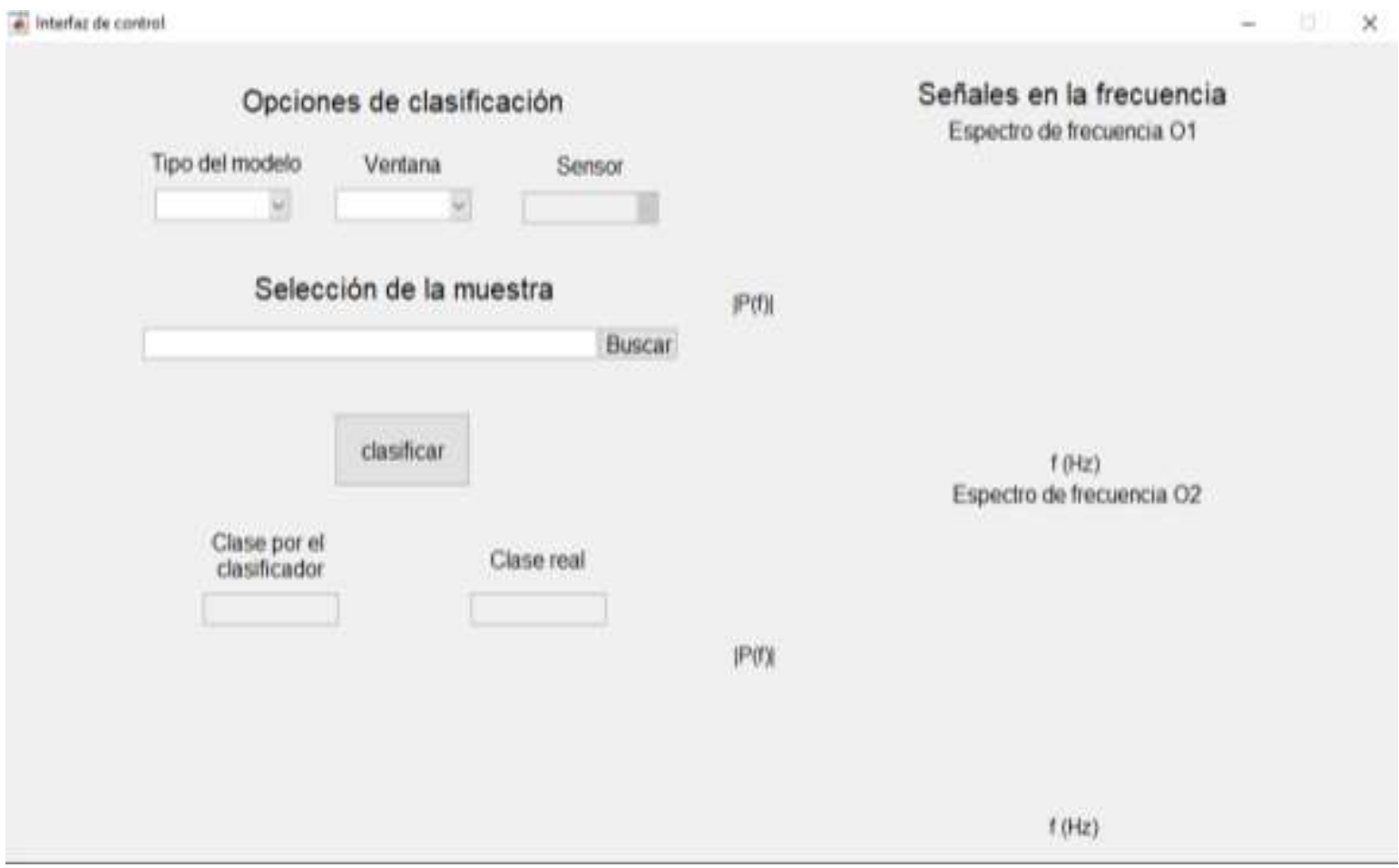

Figura 7. Interfaz gráfica de modelo de clasificador

La primera sección se llama Opciones de clasificación y tiene 3 menús de desplazamiento: Tipo de modelo, la cual da a escoger ST o SVM; Ventana, donde se define el tamaño de datos que se extraerán (20, 10 y 5 segundos); y Sensor para elegir los electrodos $O_{1} \cup O_{2}$. Este último solo se activa cuando se escoge el clasificador ST. La segunda sección se llama Selección de la muestra y se presiona el botón buscar para abrir un explorador donde se puede buscar el archivo de interés. La última sección se llama Señales en la frecuencia y se encuentra a la derecha de la interfaz. Allí se grafica $Y_{p}(k)$ a partir de los $2 \mathrm{~Hz}$ en adelante, lo cual es útil para distinguir de manera visual cual electrodo está teniendo mayor rendimiento en cuestión de la lectura de SSVEP y de esta manera, si se está usando el clasificador ST, poder escoger ese electrodo para el paso de predicción. En la parte abajo a la izquierda de la interfaz se tienen 3 componentes que dan información de la señal adquirida.

El nombre de la clase definida por el clasificador se envía a través de comunicación serial hacia el programa hecho en C\#; se realizó de esta manera por ser la forma más sencilla de comunicar Matlab con Visual Studio. El programa en $\mathrm{C \#}$ solamente requiere el nombre de la clase para manejar el comportamiento del deletreador. 


\section{RESULTADOS}

\subsection{Desempeño de los clasificadores}

Para conocer el desempeño de los clasificadores se obtuvieron las tasas de acierto como en (Friman, Volosyak, \& Gräser, 2007), para cada uno de los sujetos con los 3 tamaños de ventanas; posteriormente estas tasas serán desglosadas en matrices de confusión. En el caso del sujeto de prueba se obtuvo únicamente el desempeño para las ventanas de 10 y 5 segundos puesto que las señales de 20 segundos fueron utilizadas para el desarrollo de los clasificadores.

En la Tabla 3 están los resultados del sujeto 1. Se escribe "NA" en la columna de los 20 segundos porque estas fueron las muestras con las que se desarrollaron los clasificadores. Como análisis de los resultados se puede decir que la clasificación de las características del electrodo $\mathrm{O} 2$ con el clasificador ST tuvo mejor desempeño que con el electrodo O1, llegando alcanzar hasta un $89.17 \%$ de acierto en un tamaño de señal de 10 segundos, por lo cual éste es el que se usa en la ejecución de la aplicación. El clasificador SVM tuvo porcentajes más altos que el ST para el electrodo 01 pero menor que el de O2.

Tabla 3. Porcentajes de acierto del sujeto 1.

\begin{tabular}{|l|l|l|l|}
\hline Clasificador & \multicolumn{3}{|c|}{ Porcentaje de acierto según tamaño de la muestra } \\
\hline & 20 segundos & 10 segundos & 5 segundos \\
\hline Simple Tree O1 & NA & $84.17 \%$ & $77.50 \%$ \\
\hline Simple Tree O2 & NA & $89.17 \%$ & $82.50 \%$ \\
\hline Support Vector Machine & NA & $85.83 \%$ & $78.33 \%$ \\
\hline
\end{tabular}

En la Tabla 4 están las tasas de acierto del sujeto 2, las tasas de acierto entre la clasificación con ST para $\mathrm{O} 1$ y O2 son iguales a los 20 segundos, pero para las demás ventanas se obtuvo mejor resultado para las $f_{o 2}$. En esta tabla se observa que solo la tasa de acierto del clasificador SVM presentó un desempeño similar que el $\mathrm{O} 2$ excepto en la ventana de 5 segundos, en la cual el desempeño fue menor.

Tabla 4. Porcentajes de acierto del sujeto 2

\begin{tabular}{|l|l|l|l|}
\hline Clasificador & \multicolumn{3}{|l|}{ Porcentaje de acierto según tamaño de la muestra } \\
\hline & 20 segundos & $\begin{array}{l}10 \\
\text { segundos }\end{array}$ & 5 segundos \\
\hline Simple Tree O1 & $90.83 \%$ & $70.00 \%$ & $54.17 \%$ \\
\hline Simple Tree O2 & $90.83 \%$ & $77.50 \%$ & $70.00 \%$ \\
\hline $\begin{array}{l}\text { Support Vector } \\
\text { Machine }\end{array}$ & $90.83 \%$ & $77.50 \%$ & $63.33 \%$ \\
\hline
\end{tabular}


En la Tabla 5 se tienen los porcentajes de acierto del sujeto número 3, que son los más altos comparándolos con los resultados de los sujetos 1, 2 y 4 (tablas 2 , 3 y 5), esta tabla muestra porcentajes hasta de $94.16 \%$ de acierto en tamaños de muestra de 5 segundos en el electrodo 01 , este porcentaje hace viable utilizar este tamaño de señales dentro de la aplicación.

Tabla 5. Porcentajes de acierto del sujeto 3

\begin{tabular}{|l|l|l|l|}
\hline Clasificador & \multicolumn{3}{|l|}{ Porcentaje de acierto según tamaño de la muestra } \\
\hline & 20 segundos & $\begin{array}{l}10 \\
\text { segundos }\end{array}$ & 5 segundos \\
\hline Simple Tree O1 & $99.17 \%$ & $97.50 \%$ & $94.17 \%$ \\
\hline Simple Tree O2 & $97.50 \%$ & $90.00 \%$ & $75.83 \%$ \\
\hline $\begin{array}{l}\text { Support Vector } \\
\text { Machine }\end{array}$ & $97.50 \%$ & $90.83 \%$ & $76.67 \%$ \\
\hline
\end{tabular}

En la Tabla 6 están los porcentajes del sujeto número 4. En este sujeto la diferencia de porcentajes entre la clasificación con ST de ambos electrodos es más del $25 \%$, por lo cual si se pretende utilizar uno de los 2 electrodos, el 01 es la mejor opción. Las tasas del clasificador SVM son casi igual de bajas que el del ST O2 por lo cual en este caso solo es viable utilizar ST para el electrodo 01.

Tabla 6. Porcentajes de acierto del sujeto 4

\begin{tabular}{|l|l|l|l|}
\hline Clasificador & \multicolumn{3}{|l|}{ Porcentaje de acierto según tamaño de la muestra } \\
\hline & 20 segundos & $\begin{array}{l}10 \\
\text { segundos }\end{array}$ & 5 segundos \\
\hline Simple Tree O1 & $96.67 \%$ & $86.67 \%$ & $75.83 \%$ \\
\hline Simple Tree O2 & $72.50 \%$ & $58.33 \%$ & $41.67 \%$ \\
\hline $\begin{array}{l}\text { Support Vector } \\
\text { Machine }\end{array}$ & $75.83 \%$ & $59.17 \%$ & $45.83 \%$ \\
\hline
\end{tabular}

De acuerdo a los resultados registrados, el porcentaje promedio de acierto del ST para una señal de $10 \mathrm{~s}$ fue de $84.58 \%$ para el canal O1 y un $78.75 \%$ para el O2; mientras que el del SVM fue $78.33 \%$.

\subsection{Confusión de clases}

Para analizar la forma en que se distribuye el error en la clasificación de las clases, se generaron matrices de confusión para conocer cómo se distribuye el error en la clasificación en cada sujeto. La Figura 8 muestra las matrices de confusión de los 4 sujetos de forma promediada. En las matrices de confusión de simple tree los porcentajes de clasificación son iguales o mayores a $74.4 \%$, 
mientras que en las matrices de SVM si hay tasas menores a $74.4 \%$, en ambos casos no se llega al $100 \%$.

a)

\begin{tabular}{|c|c|c|c|c|}
\hline \multicolumn{5}{|c|}{ Simple Tree con tamaño de muestra de 5 segundos } \\
\hline \multirow{2}{*}{ Derecha } & 26.00 & 1.00 & 1.67 & 1.33 \\
\hline & $86.7 \%$ & $3.3 \%$ & $5.6 \%$ & $4.4 \%$ \\
\hline \multirow{2}{*}{ Izquierda } & 2.00 & 22.33 & 2.33 & 3.33 \\
\hline & $6.7 \%$ & $74.4 \%$ & $7.8 \%$ & $11.1 \%$ \\
\hline \multirow{2}{*}{ Abajo } & 2.00 & 1.67 & 25.00 & 1.33 \\
\hline & $6.7 \%$ & $5.6 \%$ & $83.3 \%$ & $4.4 \%$ \\
\hline \multirow{3}{*}{ Arriba } & 2.33 & 2.67 & 2.33 & 22.67 \\
\hline & $7.8 \%$ & $8.9 \%$ & $7.8 \%$ & $75.6 \%$ \\
\hline & Derecha & zquierd & Abajo & Arriba \\
\hline
\end{tabular}

Simple Tree con tamaño de muestra de 10 segundos

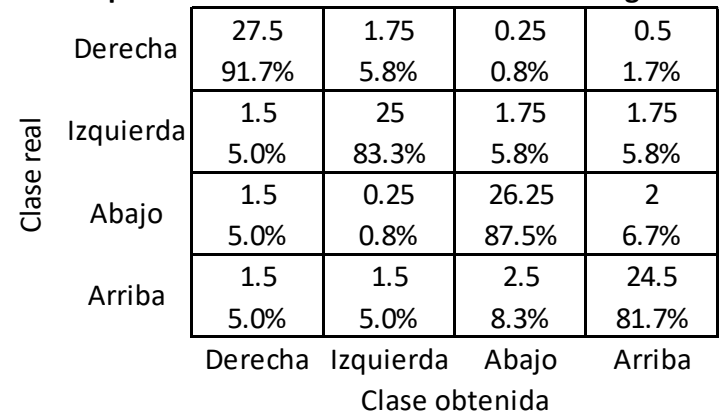

Simple Tree con tamaño de muestra de 20 segundos

\begin{tabular}{|c|c|c|c|c|}
\hline \multirow{2}{*}{ Derecha } & 29.25 & 0.25 & 0 & 0.5 \\
\hline & $97.5 \%$ & $0.8 \%$ & $0 \%$ & $1.7 \%$ \\
\hline \multirow{2}{*}{ Izquierda } & 0.25 & 29 & 0 & 0.75 \\
\hline & $0.8 \%$ & $96.7 \%$ & $0 \%$ & $2.5 \%$ \\
\hline \multirow{2}{*}{ Abajo } & 1 & 0 & 27.5 & 1.5 \\
\hline & $3.3 \%$ & $0 \%$ & $91.7 \%$ & $5.0 \%$ \\
\hline \multirow{2}{*}{ Arriba } & 1.5 & 0.25 & 1.25 & 27 \\
\hline & $5.0 \%$ & $0.8 \%$ & $4.2 \%$ & $90.0 \%$ \\
\hline & Derech & zquierd & Abajo & Arriba \\
\hline
\end{tabular}

b)

\begin{tabular}{|c|c|c|c|c|}
\hline \multicolumn{5}{|c|}{ SVM con tamaño de muestra de 5 segundos } \\
\hline \multirow{4}{*}{ Izquierda } & 22.67 & 3.33 & 1.00 & 3.00 \\
\hline & $75.6 \%$ & $11.1 \%$ & $3.3 \%$ & $10 \%$ \\
\hline & 2.33 & 19.67 & 1.67 & 6.33 \\
\hline & $7.8 \%$ & $65.6 \%$ & $5.6 \%$ & $21.1 \%$ \\
\hline \multirow{2}{*}{ Abajo } & 1.67 & 7.33 & 16.67 & 4.33 \\
\hline & $5.6 \%$ & $24.4 \%$ & $55.6 \%$ & $14.4 \%$ \\
\hline \multirow{3}{*}{ Arriba } & 3.00 & 6.67 & 5.00 & 15.33 \\
\hline & $10.0 \%$ & $22.2 \%$ & $16.7 \%$ & $51.1 \%$ \\
\hline & Derecha & zquierd & Abajo & Arriba \\
\hline
\end{tabular}

\begin{tabular}{|c|c|c|c|c|}
\hline \multicolumn{5}{|c|}{ SVM con tamaño de muestra de 10 segundos } \\
\hline \multirow{4}{*}{ Izquierda } & 26 & 2.75 & 0.25 & 1 \\
\hline & $86.7 \%$ & $9.2 \%$ & $0.8 \%$ & $3.3 \%$ \\
\hline & 2.5 & 21.75 & 2.5 & 3.25 \\
\hline & $8.3 \%$ & $72.5 \%$ & $8.3 \%$ & $10.8 \%$ \\
\hline \multirow{2}{*}{ Abajo } & 0.5 & 5.5 & 21.25 & 2.75 \\
\hline & $1.7 \%$ & $18.3 \%$ & $70.8 \%$ & $9.2 \%$ \\
\hline \multirow{2}{*}{ Arriba } & 1.25 & 2.75 & 3.25 & 22.75 \\
\hline & $4.2 \%$ & $9.2 \%$ & $10.8 \%$ & $75.8 \%$ \\
\hline & Derecha & Izquierd & Abajo & Arriba \\
\hline
\end{tabular}

\begin{tabular}{|c|c|c|c|c|}
\hline \multicolumn{5}{|c|}{ SVM con tamaño de muestra de 20 segundos } \\
\hline \multirow{2}{*}{ Derecha } & 28 & 1 & 0 & 1 \\
\hline & $93.3 \%$ & $3.3 \%$ & $0 \%$ & $3.3 \%$ \\
\hline \multirow{2}{*}{ Izquierda } & 0.5 & 28 & 0 & 1.5 \\
\hline & $1.7 \%$ & $93.3 \%$ & $0 \%$ & $5.0 \%$ \\
\hline \multirow{2}{*}{ Abajo } & 0.75 & 2 & 24.75 & 2.5 \\
\hline & $2.5 \%$ & $6.7 \%$ & $82.5 \%$ & $8.3 \%$ \\
\hline \multirow{3}{*}{ Arriba } & 0.75 & 2.75 & 2.25 & 24.25 \\
\hline & $2.5 \%$ & $9.2 \%$ & $7.5 \%$ & $80.8 \%$ \\
\hline & Derecha & zquierd & Abajo & Arriba \\
\hline
\end{tabular}

Figura 8. Promedio de las matrices de confusión de los 4 sujetos a) ST b) SVM.

Para todos los sujetos y clasificadores, la clase Arriba obtuvo el mayor error y la clase Derecha el mayor acierto. Como era de esperarse, el tamaño de la señal afectó proporcionalmente la tasa de acierto de la clasificación, teniendo los mejores porcentajes en las señales de 20 segundos y los peores a los 5 segundos.

El desempeño entre sujetos fue diferente principalmente por el hecho de que no todos prestan el mismo nivel de atención durante la sesión, o porque el individuo puede encontrarse fatigado e incluso, el manejo de la diadema en cuestión de la posición e impedancia de los electrodos sobre el cráneo puede 
afectar el experimento. Por estas razones no se puede esperar un desempeño igual entre todos los sujetos.

\section{CONCLUSIONES}

De acuerdo con los resultados experimentales, los porcentajes de una correcta clasificación son más altos entre más grande sea el tamaño de la muestra ya que la probabilidad de que se genere un SSVEP aumenta. El tamaño de las muestras de 20 segundos tuvo un porcentaje de acierto bastante alto (de más del $90 \%$ para el ST), sin embargo, este tamaño de señal es muy poco práctico para la creación de un sistema $\mathrm{BCl}$ porque mirar un estímulo por 20 segundos puede resultar muy lento y agotador para la mayoría de las personas, por lo cual se hicieron clasificadores también para tiempos de 10 y 5 segundos. En los tiempos de 10 segundos sí se tuvo un porcentaje aceptable, pero a los 5 segundos el error ya fue considerable. Un sistema BCI basado en SSVEP debe tener tamaños de muestra de máximo 5 segundos para que sea práctico.

Hablando de los clasificadores, un clasificador SVM tiene tasas de acierto mejores a la clasificación de Simple Tree del electrodo con menor desempeño, la ventaja del SVM radica en que no es necesario saber en cual electrodo se está generando un SSVEP con mayor amplitud, pero una vez se conozca este electrodo, es mejor utilizar el clasificador Simple Tree porque tiene un porcentaje de clasificación mayor que el SVM, esto se debe a que el SVM combina ambos electrodos por lo que el desempeño combinado de ambos electrodos se ve grandemente influenciado por el electrodo con peor desempeño.

Las muestras que fueron clasificadas erróneamente se debió a que no se generó un SSVEP, o si se generó, fue con una amplitud muy pequeña que no fue posible distinguir dentro de la señal de en el dominio de la frecuencia, por lo que al buscar la amplitud máxima dentro de la función, se escoge otra que no corresponde a una señal de SSVEP.

\section{AGRADECIMIENTOS}

Se agradece al Tecnológico Nacional de México por el apoyo brindado para la realización de este trabajo bajo el proyecto 5684.16-P

\section{REFERENCIAS}

Abbasi, M. A., Gaume, A., Francis, N., Dreyfus, G., \& Vialatte, F. B. (2015). Fast calibration of a thirteen-command $\mathrm{BCI}$ by simulating SSVEPs from trains of 
transient VEPs - Towards time-domain SSVEP BCI paradigms. Conferencia internacional de ineniería neural. Montpellier.

Bondre, C., \& Kapgate, D. (2015). Steady State Visually Evoked Potentials Framework in Brain Computer Interface. International Journal of Advanced Technology in Engineering and Science, 3(1), 167-175.

Cheng, M., Gao, X., Gao, S., \& Xu, D. (oct 2002). Design and Implementation of a Brain-Computer Interface With High Transfer Rates. IEEE Transactions on biomedical engineering, 49(10), 1181-1186.

Cotrina, A., Benevides, A. B., Castillo-Garcia, J., \& Benevides, A. B. (julio 2017). A SSVEP-BCI Setup Based on Depth-of-Field. IEEE transactions on neural systems and rehabilitation engineering, 25(7), 1047-1057.

da Cruz, J. N., Wan, F., Wong, C. M., \& Cao, T. (2015). Adaptive time-window length based on online performance measurement in SSVEP-based BCls. Neurocomputing, 149, 93-99.

Ding, J., Sperling, G., \& Srinivasan, R. (2006). Attentional modulation of SSVEP power depends on the network tagged by the flicker frequency. Cerebral cortex, 16(7), 1016-1029.

Friman, O., Volosyak, I., \& Gräser, A. (2007). Multiple channel detection of steady-state visual evoked potentials for brain-computer interfaces. IEEE transactions on bio-medical engineering, 54.

Funase, A., Wakita, K., Ita, A., \& Takumi, I. (2016). SSVEP by checkerboard related to grid size and board size. Conferencia anual de la asociación del procesamiento de la información y señales. Hong Kong.

Ji, H., Li, J., Lu, R., Gu, R., Cao, L., \& Gong, X. (2016). EEG Classification for Hybrid Brain-Computer Interface Using a Tensor Based Multiclass Multimodal Analysis Scheme. Computational Intelligence and Neuroscience, 2016(1), 1-15.

Lee, C.-C., Chuang, C.-C., Yeng, C.-H., Chen, Y.-J., \& Lin, B.-S. (Dic 2017). Noise Suppression by Minima Controlled Recursive Averaging for SSVEPBased BCls With Single Channel. IEEE Signal processing letters, 24(12), 17831787.

Lee, Y.-C., Lin, W.-C., Cherng, F.-Y., \& Ko, L.-W. (2016). A Visual Attention Monitor Based on Steady-State Visual Evoked Potential. IEEE Transactions on neural systems and rehabilitation engineering, 24(3), 399-408.

Lim, J.-H., Lee, J.-H., Hwang, H.-J., Kim, D. H., \& Im, C.-H. (2015). Development of a hybrid mental spelling system combining SSVEP-based brain-computer interface and webcam-based eye tracking. Biomedical Signal Processing and Control, 21(1), 99-104. 
Maye, A., Zhang, D., \& Engel, A. K. (julio 2017). Utilizing Retinotopic Mapping for a Multi-Target SSVEP BCI With a Single Flicker Frequency. IEEE Transations on neural systems and rehabilitation engireering, 25(7), 1026-1036.

Mitchell, T. M. (1997). Machine learning. WCB/McGraw-Hill.

Nakanishi, M., Y. W., Hsu, S.-H., Wang, Y.-T., \& Jung, T.-P. (sep 2017). Independent Component Analysis-Based Spatial Filtering Improves TemplateBased SSVEP Detection. Conferencia internacional anual en ingeniería en medicina y sociedad de biología. Seogwipo.

Shyam Prasad, P. M., Swarnkar, R., Visnu Prasad, K. V., Radhakrishnan, M., Hashmi, M. F., \& Keskar, A. G. (2017). SSVEP signal detection for BCl application. Proceedings - 7th IEEE International Advanced Computing Conference, IACC 2017, 590-595.

Tang, Z., Wang, Y., Dong, G., Pei, W., \& Chen, H. (2017). Learning to control an SSVEP-based $\mathrm{BCl}$ speller in naïve subjects. Conferencia internacional de ingeniería en medicina y sociedad de biología. Seogwipo.

Trujillo, J. M., \& Borrego, C. J. (1985). Potenciales Evocados Visuales. Acta Médica Colombiana, 10(3), 113-124.

Turnip, A., Suhendra, M. A., K, D. E., \& Sihombing, P. (2017). Design of Extraction Method of SSVEP Brain Activity with IIR Chebyshev. Conferencia internacional de intrumentación, control y automatización. Yogyakarta.

Yin, E., Zhou, Z., Jiang, J., Yu, Y., \& Hu, D. (junio 2015). A Dynamically Optimized SSVEP Brain-Computer Interface $(\mathrm{BCl})$ Speller. IEEE Transactions on Biomedical Engineering, 62(6), 1447-1456.

Zhang, N., Yu, Y., Yin, E., \& Zhou, Z. (2016). Performance of virtual stimulus motion based on the SSVEP-BCI. IEEE International Symposium Computer, Consumer and Control. Xian.

Zhanga, Y., Zhou, G., Jin, J., Wanga, X., \& Cichockib, A. (2015). SSVEP recognition using common feature analysis in brain-computer nterface. Journal of Neuroscience Methods, 244(1), 8-15.

Zhao, J., Li, W., Mao, X., Hu, H., Niu, L., \& Chen, G. (Junio 2017). BehaviorBased SSVEP Hierarchical Architecture for Telepresence Control of Humanoid Robot to Achieve Full-Body Movement. IEEE Transactions on cognitive and developmental system, 9(2), 197-209.

Zhao, X., Chu, Y., Han, J., \& Zhang, Z. (julio 2016). SSVEP-Based BrainComputer Interface Controlled Functional Electrical Stimulation System for Upper Extremity Rehabilitation. IEEE Transactions on systems, man and cybernetics: systems, 46(7), 947-956. 


\section{Notas Biográficas}

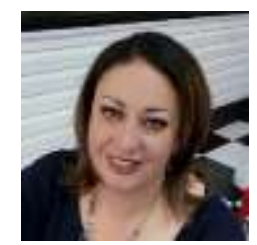

Alma Delia Corral Sáenz recibió el título de Ingeniera en Sistemas Computacionales en Hardware de la Universidad Autónoma de Chihuahua en 1999 y el de Maestra en Ciencias en Ingeniería Electrónica del Instituto Tecnológico de Chihuahua en 2003. Actualmente es profesora y coordinadora del Doctorado y la Maestría en Ciencias en Ingeniería Electrónica en el mismo Instituto, y participa en trabajos de investigación de las áreas de procesamiento de señales y visión por computadora.

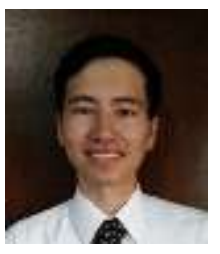

Raúl Rangel González recibió el título de Ingeniero en Electrónica en 2017 del Instituto Tecnológico de Chihuahua y actualmente es estudiante de la Maestría en Ciencias en Ingeniería Electrónica en el mismo Instituto, donde trabaja en proyectos relacionados al procesamiento y análisis de señales SSVEP en el laboratorio de Percepción Visual. Sus intereses de investigación incluyen procesamiento de señales EEG, visión por computadora, procesamiento de imágenes y video, percepción visual e inteligencia computacional.

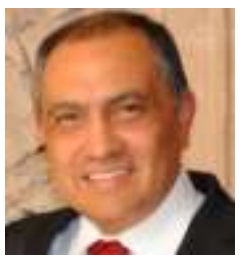

Mario Ignacio Chacón Murguía recibió el título de Ingeniero Industrial en Electrónica y el de Maestro en Ciencias en Ingeniería Electrónica en 1982 y 1985 respectivamente del Instituto Tecnológico de Chihuahua, y el grado de Doctor en Filosofía en Ingeniería Eléctrica de New Mexico State University EEUU en 1998. Actualmente es Profesor - Investigador en el Instituto Tecnológico de Chihuahua y director del laboratorio de Percepción Visual. Ha desarrollado 44 proyectos para varias compañías e instituciones. Cuenta con más de 175 publicaciones en revistas y congresos nacionales e internacionales, así como tres libros publicados. Ha dirigido 72 tesis de ingeniería, maestría y doctorado. Su investigación actual es sobre visión por computadora, y procesamiento de imágenes y señales utilizando inteligencia computacional. El Dr. Chacón es miembro Senior de la IEEE y miembro de las sociedades IEEE Computational Intelligence, IEEE Digital Signal Processing y miembro del SNI.

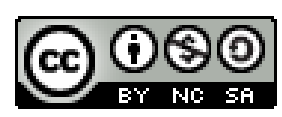

Esta obra está bajo una licencia de Creative Commons Reconocimiento-NoComercial-Compartirlgual 2.5 México. 